\title{
The Lie transformation group model of visual perception
}

\author{
PETER C. DODWELL \\ Queen's University, Kingston, Ontario, Canada
}

\begin{abstract}
The Lie transformation group model of neuropsychology (LTG/NP) purports to represent and explain how the locally smooth processes observed in the visual field, and their integration into the global field of visual phenomena, are consequences of special properties of the underlying neuronal complex. These properties are modeled by a specific set of mathematical structures that account both for local (infinitesimal) operations and for their generation of the "integral curves" that are visual contours. The purpose of this tutorial paper is to expound, as nontechnically as possible, the mathematical basis for LTG/NP, and to evaluate that model against a reasonable set of criteria for a neuropsychological theory. It is shown that this approach to spatial vision is closer to the mainstream of current theoretical work than might be assumed; recent experimental support for LTG/NP is described.
\end{abstract}

\section{INTRODUCTION}

In recent years, a variety of mathematical models have been proposed as theoretical tools for the analysis and understanding of perceptual processes. Such models differ in many ways, for example, in the sort of mathematics involved, type of implementation, abstractness, and degree of generality. Among these, geometrical models have not been prominent, although they would seem to offer the ideal vehicle for expressing the essential nature of spatial vision, and pattern recognition in particular (Dodwell, 1982). The purpose of this paper is to introduce and discuss a geometrically based model that, if valid, has great potential for deepening our understanding of perceptual processes. I say "introduce" advisedly; although the model has been in existence for well over a decade, it has attracted little attention and, until quite recently, has not led to any specifically new lines of experimental research. Why this should be so we shall shortly see. The model is the Lie transformation group model of neuropsychology, or LTG/NP.

The model warrants serious attention because it claims both to represent and explain a detailed and specific set of relationships between physiology and perceptual phenomena, on the one hand, and to bridge a major theoretical gap between local processes of coding and global processes of pattern synthesis, on the other.

In 1966, W. C. Hoffman published a paper showing how the mathematical theory of continuous

\footnotetext{
The final preparation of this paper was made during tenure of a Killam Research Fellowship. This support is gratefully acknowleged. The author's address is: Department of Psychology, Queen's University, Kingston, Ontario K7L 3N6, Canada.
}

transformation groups could be applied to visual space perception and pattern recognition (Hoffman, 1966). Since then, the model has been revised and considerably extended, to apply not only to elementary aspects of vision, but also to most forms of brain function. ${ }^{1}$ The original paper was technically too difficult for most psychologists to comprehend, being couched in a mathematical terminology and invoking concepts with which most of us (and indeed many mathematicians) are unfamiliar. Subsequent publications (e.g., Hoffman, 1970, 1977, 1980) have both broadened and deepened the ideas, and also drawn on ever higher and more abstract levels of mathematical theory. While the mathematical treatment is both technical and refined, the ideas underlying it are not in themselves difficult to understand. The main purpose of the present paper is to expound the elementary part of the theory in a form that should make it accessible to psychologists by explaining the nature of the mathematical concepts and operations involved. A second and, to my mind, equally important aim is to show how the model relates very directly to some contemporary notions about transformation and invariance under transformation. In this sense, Hoffman's ideas are much closer to the mainstream of perceptual theorizing than has generally been realized.

Hoffman (1977) coined the phrase "Lie Transformation Group Approach to Neuropsychology" (LTG/NP) for his model. Sophus Lie was the Norwegian mathematician who, late in the 19th century, developed the theory of continuous transformation groups. The LTG/NP model has, until recently, had remarkably little impact in psychology, almost certainly because the level of mathematical knowledge required to understand even the first paper is fairly 
high. However, within the last few years several experimental papers have appeared which test various aspects of LTG/NP (see Section VIII below). This trend will no doubt continue, and so it seems desirable to make available an exposition of the model that describes its basic nature without too much technical detail. While this presentation will be restricted to the very basic elements, which I shall call the "primitive model," a recent statement of LTG/NP, together with critiques and summaries of relevant experimental work, is to be found in Paillard (1977), which is the best comprehensive introduction to this approach to visual processing.

In general, it can be said that LTG/NP exploits the geometrical character of both the visual field and the neural machinery of the retina, retinocortical pathways, and visual cortex (Dodwell, 1982; Hoffman \& Dodwell, Note 1). It seeks to relate microgenetic processes in the visual field, which are reflected in strictly localized activities within the nervous system (activity of individual neurons) to more macroscopic aspects of both the visual scene and the neural activities which underlie pattern processing. Thus, while the model is couched in abstract terms, the interpretation postulated for it, both psychophysically and neuropsychologically, is quite immediate.

The plan of the paper is to describe first the mathematical background needed to comprehend the nature of the model. This is done as straightforwardly as possible, only some basic elements of algebra, coordinate geometry, and the first steps in differential calculus being assumed. Secondly, the interpretation of these mathematical ideas in the LTG/NP model is introduced, together with discussion of the neuropsychological processes claimed to be the substrate of perceptual events. This is the heart of the matter, as LTG/NP's claim to be worthy of serious attention is based on the postulate that mathematical "operators" of the model are to be identified as the formal equivalents of elements within already-known anatomical structures of the visual nervous system whose physiological activities are-at least in part-well understood.

In the final sections, I shall discuss the sorts of evidence that are claimed to support the model, outline some of the new experimental findings gathered explicitly within the framework it provides, and evaluate it briefly as a neuropsychological theory.

\section{MATHEMATICAL PROLEGOMENA}

\section{What Is a Continuous Transformation Group?}

We start with the basically simple concept of a transformation. A transformation is an operation taking some geometric object, such as a point, line, or figure, into some other (usually similar) object, the simplest such operation being a translation, say along a horizontal line. A continuous transformation does this operation smoothly (continuously) by a series of infinitesimally small steps "glued together" locally in some fashion. A transformation group is a set of transformations having the mathematical properties of a group, namely that any two transformations successively applied are themselves a transformation, there is an inverse (canceling) transformation, and an identity (null) transformation. A series of transformations can be applied in any way, so long as order is preserved; the group is said to be "associative." Example: Translations of a point along a line in either direction satisfy these conditions. A continuous transformation group is one in which the transformations are performed smoothly (continuously) rather than in discrete steps. Example: The operations of sliding a point along a line smoothly so that it traverses all intermediate points between its starting and final positions satisfy these conditions. Similarly, the smooth rotations of a line about a point would form a continuous transformation group because each rotation $\theta \mathrm{deg}$, of any magnitude, can be canceled by rotation $-\theta$ deg (a rotation of $0 \mathrm{deg}$ is the "null" transformation), and rotations can be added (and subtracted) to give new rotations. In contrast, the rotations of an equilateral triangle into it-

TRANSFORMATION: EXAMPLE IS TRANSLATION

A

B

CONLINUOUS IRANSFORMATION: SMOOTH TRANSLATION,

TRAVERSING ALL INTERMEDIATE POINTS,

ANOTHER EXAMPLE: ROTATIONS OF LINE ABOUT POINT.

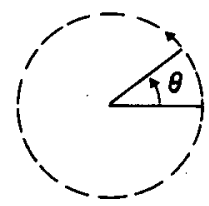

PER CONTRA: DISCRETE IRANSFORMAIION GROUP:

ROTATIONS OF AN EQUILATERAL TRIANGLE

INTO ITSELF,

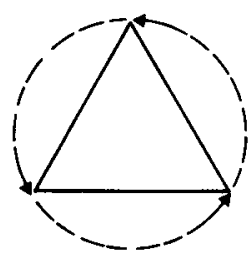

Figure 1. Examples of continuous and discontinuous transformation groups. In the former, the transformation process is by a sequence of (infinitesimally) small steps, whereas in the latter the transformation is a discrete, step function. 
self about its center would be a discontinuous group; only rotations $n \times 120^{\circ}(n= \pm 0,1,2 \ldots)$ would count as transformations within the group, and these are clearly discrete steps. In any case, the characteristic of a group is that the operation(s) allowed do(es) not lead to any new sorts of state or entity: the group has a self-consistent, enclosed quality; mathematicians say it exhibits "closure."

While Lie's theory of continuous transformation groups is based on these rather straightforward ideas, it explores their ramifications into highly abstract realms. We shall be concerned only with the very basic types of continuous, or Lie, transformation groups and their elementary applications. Even so, a certain level of mathematical understanding is required to grasp the significance of the approach; I shall assume an understanding of the concepts of such simple functions as $y=f(x)$, and $F(x, y)=0$, and basic differentiation,

$$
\frac{d}{d x}[f(x)]=\frac{d}{d x}(y)=\frac{d y}{d x}
$$

Several specific geometric entities which need explicit description are presumed to underlie the action of Lie transformation groups, the first of these being a manifold.

\section{What Is a Manifold?}

Basically, a manifold is a surface; in the case of a two-dimensional manifold, the intuitive notion of a smooth surface (one without cuts or holes in it) fairly adequately defines the concept. Although a manifold may have an arbitrarily high number of dimensions, we shall be concerned only with the concept of a twodimensional manifold, and one that is differentiablethat is, a manifold on which smooth, continuous operations can be performed, like drawing with the head of a pencil across a sheet of paper. A manifold can have associated with it one or more vectorfields, which are the agents mediating locally such smooth operations.

\section{What Is a Vectorfield?}

A (bound) vector is a directed line carrying information about position, direction, and magnitude, or strength. A familiar example is the tangent vector to a point moving on a smooth curve (or trajectory) with a given velocity; in this case, the tangent vector to the curve at that point gives the slope, or the instantaneous velocity with which the point is moving (see Figure 2a). Conversely, it is possible to think of a sequence of tangent vectors generating (successively in time) the path along which the point is moving. This idea is also illustrated in Figure $2 b$. Think of a ball floating down a river, whose surface is clearly a type of manifold; at any instant in time, the ball's
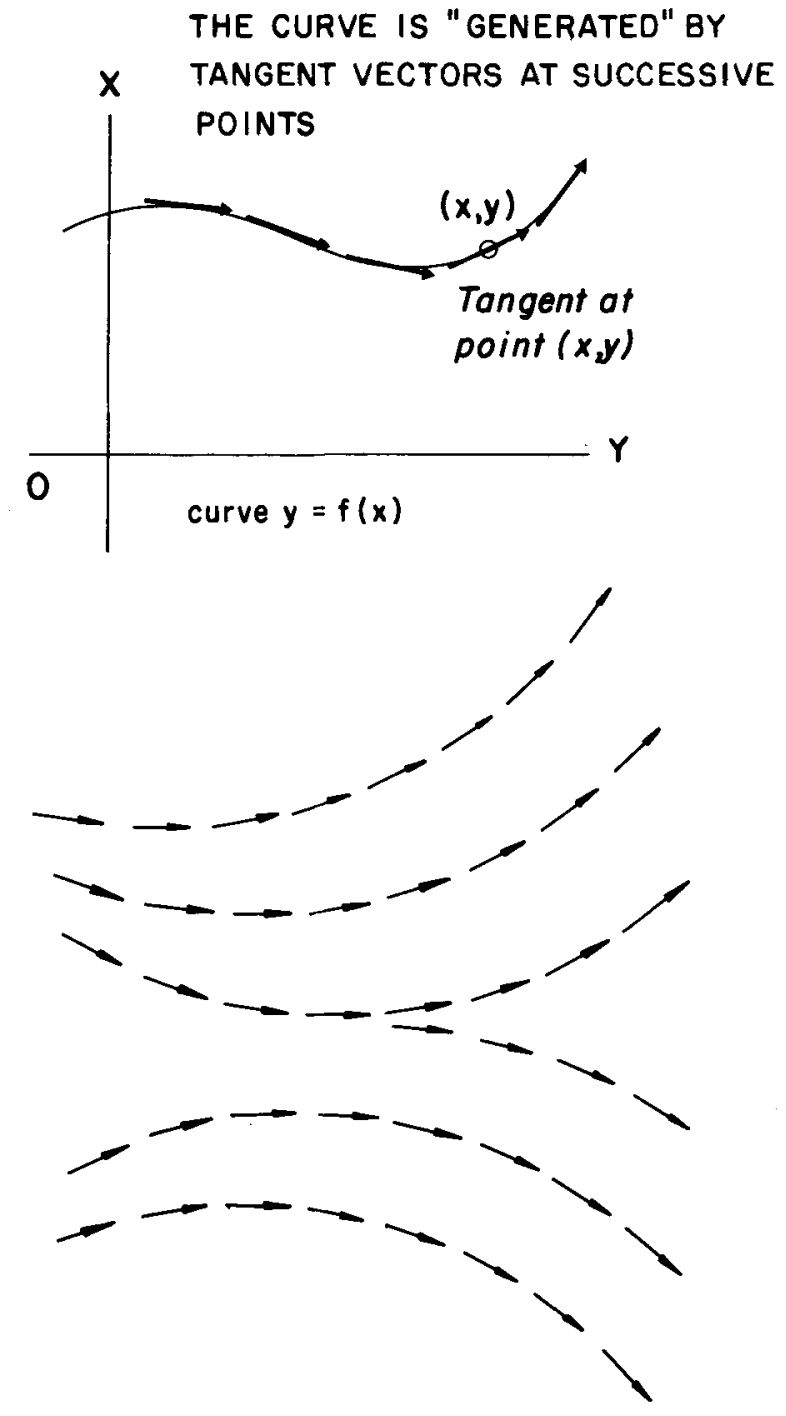

Vectors generate orbits (contours)

Figure 2. (a) Illustration of the concept of a smooth curve (function) in the plane, and its tangent vector at a point; the functoh can be generated by a sequence of tangents at (Infinitesimally) close positions along the curve. (b) Sequences of vectors, lined up head-to-tall, generate smooth ("integral") curves in the plane.

motion (speed and direction) could be expressed as a vector. This is the local, instantaneous characterization of the ball's path; the vector will change over time as the ball moves. One could describe the path of the ball in terms of those successively changing vectors. But there are many paths which the ball could take, depending on the point at which it is dropped into the river, and on the pattern and direction of flow of the river's current. Thus, there are potentially many different paths which could be given this vectorial representation, and their com- 
plete set would constitute a vectorfield. More formally, a vectorfield is the set of vectors associated with all points on a surface or manifold, which potentially can generate all possible paths or trajectories on that manifold. It is intuitively clear from the river example that the sorts of path that can be traced out will depend on the characteristics, or structure, of the manifold on which the vectorfield operates. To correct the possible misapprehension that a vector or vectorfield has to be associated with real movement, the example of a magnetic field of force is appropriate; in this case, the shape and strength of the magnetic field (for example, shown by tapping iron filings on a piece of paper over the pole of a magnet) also are properly defined in terms of vectors and vectorfields. In every case, the important point is that the vectors express the local properties of the field in question. Strictly speaking, the vectors give the best local linear approximation to the field properties at any given position.

As the example in Figure 2 suggests, the vectors (and vectorfields) may be related by an equation that expresses the values of the vectors at every point in the field. If so, the vectorfield has a well-defined structure. An example using a familiar function illustrates the relationships between the vectors that are elements in a vectorfield, the structure of that field, and the equation (a so-called differential equation) that expresses vector properties as a function of their position in the field.

The equation for a circle of radius $r$, centered at the origin of coordinates, is:

$$
x^{2}+y^{2}=r^{2}
$$

In familiar terms, Equation 1 gives the locus of all points on the circle, or of all points that are exactly a distance $r$ from the origin.

Differentiating Equation 1 with respect to $x$, we obtain the expression:

$$
\frac{d}{d x}\left[x^{2}+y^{2}-r^{2}\right]=0
$$

so

$$
2 x+2 y \frac{d y}{d x}=0
$$

or

$$
\frac{d y}{d x}=-\frac{x}{y}
$$

This is a simple differential equation, and expresses the fact that for all points in the field the slope $d y / d x$ of the function (1) is $-x / y$, regardless of the value of r. A numerical example is shown in Figure 3: Obviously the structure of this vectorfield is determined by Equation 2, and we can think of the vectors as the generators of particular circles, de-
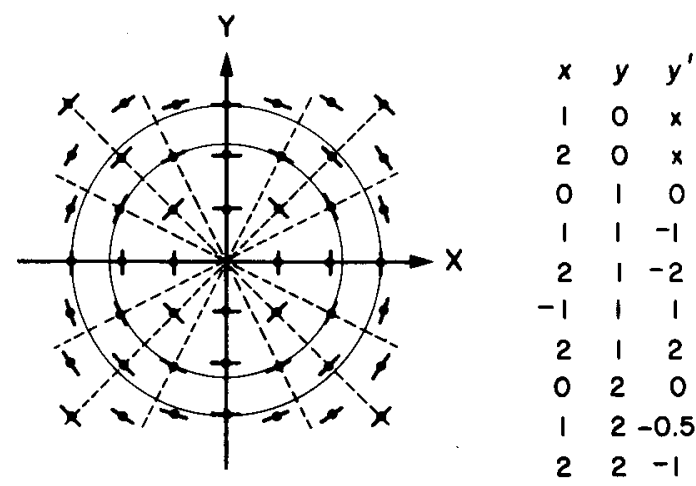

Line elements for the equation $y^{\prime}=-x / y$.

Figure 3. Numerical example of how the differential equation $(d y / d x)=(-x / y)$, generates tangent vectors to circles at all positions. The vectorfield with global circular structure is thereby generated ( $y^{\prime}$ is also used for the symbol dy/dx, as in this figure).

pending on the choice of r. Figure 4 illustrates another vectorfield and its characteristic differential equation.

Thus, we see that the concepts of vector and vectorfield nicely encompass the dual characteristics of local operations and global structure on a manifold. Not every manifold and its associated vectorfield(s) will have such simple properties as those outlined above, but these examples express the important principles involved, and provide a sufficient basis for our purposes.

\section{Lie Operators and Lie Orbits}

We turn now to the consideration of those special properties of vectorfields that constitute the basis for

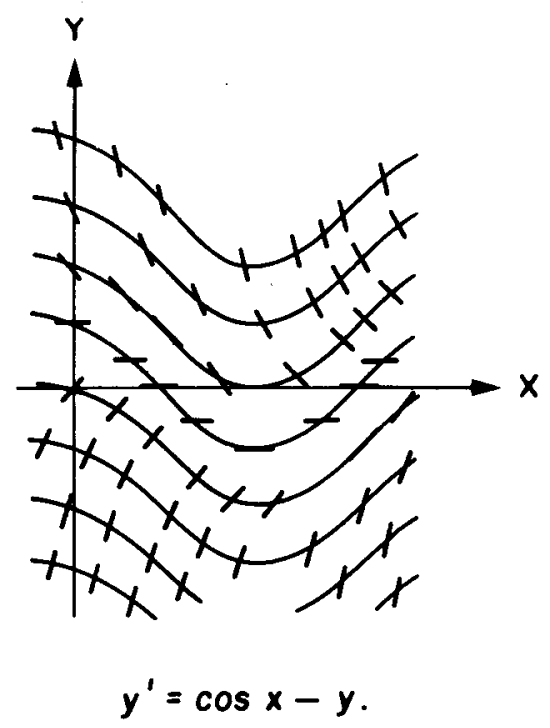

Figure 4. Another structured vectorfield and its generating differential equation, $d y / d x=\cos x-y$. 
a Lie transformation group and its implementation.

The infinitesimal (local) generator of a smooth transformation is a differential operator, or Lie operator, of the form

$$
\mathscr{L}=\left[f(x, y) \frac{\partial}{\partial x}+g(x, y) \frac{\partial}{\partial y}\right],
$$

which operates on some function, F. F is an equation, of the sort familiar in analytical geometry, which expresses a relationship between two (or more) variables. In the two-dimensional case, $F$ is, for our purposes, the equation of a simple curve or set of curves, like Equation 1. Expression 3 looks formidable, and is the statement for a general first-order two-dimensional operator, but it is, in fact, a straightforward generalization from the ideas expressed in Figure 2. The expression $d y / d x$, or $(d / d x)[f(y)]$, represents the slope of the function (Figure $2 a$ ), that is, the rate at which $y$ is changing per unit change in $x$. The expression reads: "differentiate with respect to x" and is a vector operator: it means "find the slope of the function $f(x)$ ", which is the equivalent of "find the vector that best expresses the function $\mathrm{f}(\mathrm{x})$ locally (at some point)." Or, more exactly: "find the vector that gives the best linear approximation to $f(x)$ loally." In just the same way, the Lie operator, $\mathscr{L}$ (Expression 3), is a local vector operator. The partial differential operators $\partial / \partial \mathrm{x}$ and $\partial / \partial \mathrm{y}$ take components of a vector such as $\mathrm{dy} / \mathrm{dx}$ along two orthogonal directions and can be thought of as the horizontal and vertical components of $\mathrm{dy} / \mathrm{dx}$ in Figure $2 a$. The expression $\partial / \partial x$ reads: "partial differentiation with respect to $x$, it being understood that other variables, such as $y$, are treated as constants in this operation." Similarly, $\partial / \partial y$ reads: "partial differentiation with respect to y." The expression $\mathscr{L}$ can be thought of as the general local vector operator that measures the total rate of change in some function F, expressing this in terms of two components in orthogonal directions. A fuller discussion of these ideas, one that is both elegant and straightforward, can be found in Bruter (1977).

We shall be concerned only with very simple examples of operators of the form $\mathscr{L}$. For instance, when $f(x, y)=1$ and $g(x, y)=0$ in Equation 3, we get the operator $\mathscr{L}_{\mathrm{x}}=\partial / \partial \mathrm{x}$, which is the expression for the vector which translates a point, or set of points, along or parallel to the $x$ axis; or, we can say it expresses a rate of change in the $x$ direction only. Similarly, when $f(x, y)=0$ and $g(x, y)=1$, we get the operator $\mathscr{L}_{\mathrm{y}}=\partial / \partial \mathrm{y}$, which is the generator of vertical (y axis) excursions. Repeated application of one of these operators leads to smooth translations, in the horizontal direction for $\partial / \partial x$ and in the vertical direction for $\partial / \partial y$. In general, repeated application of an operator $\mathscr{L}$ generates an orbit or trajectory, as indicated in Figure 2b, although we shall not deal with the details of mathematical integration that this involves. A basic tenet of LTG/NP is that the vectorfield operations generate, or "admit," only a restricted set of continuous transformation groups, and that these constrain visual processing in a fundamental way. The vectorfield in Figure 3 obviously "admits" rotational transformation, for example; it also "generates" circular orbits.

\section{NEUROPSYCHOLOGICAL INTERPRETATION}

Clearly, the simple and complex cortical receptive field units discovered by Hubel and Wiesel (1962) have vector-like properties, for each unit has associated with it a position, direction, and (probably) magnitude. Hoffman identifies these units as the elements of a vectorfield, or set of vectorfields, on the visual cortical manifold. The structure of that manifold will determine the nature of the vectorfields, and thus the basic paths, trajectories, or orbits that can occur and those vectorfields, in turn, control the Gestalt properties of visual space (for an elaboration of this idea, see Hoffman \& Dodwell, Note 1).

It is true that a great deal of structure (cytorarchitecture) has been identified within the visual cortex (e.g., Hubel \& Wiesel, 1977), structure that has to do with orientation columns, ocular dominance slabs, and the like. All this vast amount of new knowledge about the cortex and its organization, however, tells us extraordinarily little about how the local processes embodied in individual neuronal activity are integrated into coherent operations which generate macroscopic properties of vision. A fundamental postulate of LTG/NP is that the integrative process is best understood by treating the cortex as a manifold on which vectorfields operate, by interpreting the local vectorial operations, in terms of neural processing à la Hubel and Wiesel, and in turn to model the integrative neural action as a function of the postulated vectorfields and their global properties. The latter are embodied in the mathematical structure outlined in II3 and II4.

Invoking the concept of a vectorfield allows one to proceed naturally from a local process, the vector, embodied in individual neuronal activity, to paths or trajectories across parts or even the whole of the relevant manifold, as described in the previous section. That is the neuropsychological aspect of the theory: the perceptual aspect interprets those trajectories in some cases as visual contours or, in others, as the basis of a certain class of continuous transformations which occur naturally in the visual field. Both aspects are illustrated in the example of Figure 3, although the formal basis for the relevant class of Lie orbits has not yet been developed. The best way to do this is through closer scrutiny of the sorts of perceptual pro- 
cessing the visual system must sustain in order to be biologically efficient.

\section{PERCEPTUAL STRUCTURE: ORBITS OF THE PRIMITIVE MODEL}

\section{Translation}

It is very well known in visual science that the horizontal and vertical directions play fundamental roles both psychophysically at threshold and for pattern recognition. LTG/NP has a novel explanation of why this should be so; horizontal and vertical lines are a basic pair of Lie orbits, that is to say, they are the orbits generated by iterative action of the simplest Lie operators, $\mathscr{L}_{\text {x }}$ and $\mathscr{L}_{\text {y }}$ (section II4). But why should the Lie operators and orbits be relevant, and why should we expect them to play any role, let alone a dominant role, in visual processing? To answer this question, we must consider again the concept of transformations and the role they play in pattern processing and recognition. As J. J. Gibson (1950) so forcefully argued, as observers in a natural environment we are continually in motion, and the very fact of that motion imposes richness and variety on the visual input, or, as Gibson would say, on the optic array. Gibson was fundamentally uninterested in the internal aspects of the coding of the optic array, and that is a weakness in his approach. If we think of even the simplest self-produced movement of eye or head, there are concomitant changes in the retinal image which must have profound effects on the neural coding and representation of the array. Gibson may be right in claiming that most (if not all) the information for veridical perception is out there, but surely is wrong in ignoring the sheer extent and potential problems of computing the transformations and the invariances that are detected despite them-or perhaps, in some cases, because of them. In one sense, LTG/NP offers precisely the solution of the problem of detecting invariances under transformation that Gibson neglected, because it proposes mechanisms for computing them. ${ }^{2}$

An important aspect of visual motion and the attendant computations it implies on the "neural image" is simple translation, which may be separated into horizontal and vertical components. Because of the way we are constructed, the way we move around in the world, and the near-universal presence of gravitational cues, these components dominate translatory movement, although frequently both components will occur in conjunction with one another. At all events, $\partial / \partial x$ and $\partial / \partial y$ are the operators which most fittingly define the transformation groups of simple translation. It should be noted that these are transformations that do not normally disrupt pattern recognition (PR); that is, they are transformations under which PR is invariant. Ecologically, it is clear why this condition must hold. As the or- ganism moves around in its environment, it has to be able to ignore or cancel out such transformations. Whether engraved by evolutionary pressure or acquired through perceptual learning, the computation of these transformations, and their "cancellation," have to be fundamental properties of a coherent and stable visual processor (Dodwell, 1970).

Thus, we have arrived at the following ideas: First, we have identified a pair of transformation groups under which $P R$ is known to be stable, or invariant; they are characterized by the simple Lie operators, $\mathscr{L}_{x}$ and $\mathscr{L}_{y}$, which, in turn, generate orbits (horizontal and vertical contours-section II4). Second, if the processing of visual images under translation involves the transformation groups of $\mathscr{L}_{\mathrm{x}}$ and $\mathscr{L}_{\mathrm{y}}$, this implies a certain structure for the associated vectorfields. From this point, we infer that, if the visual cortical manifold has that structure (not to the exclusion of other structures, of course), its presence should be discernible in phenomena of spatial vision and pattern and object recognition. This is the sense in which LTG/NP explains the salient role of horizontal and vertical directions in PR.

\section{Dilation and Rotation}

Translation, of course, is not the only visual transformation under which PR must remain invariant. It must not be disrupted as objects approach or recede, and also (within limits) must remain invariant under rotations in the frontal plane.

How can these transformations be characterized, and are there Lie operators that generate them? Again following Gibson's lead, we imagine an organism locomoting straight ahead; the optical "flow" will be an expansion along lines emanating from a point at the straight-ahead position (see Figure 5). The Lie operator that generates such an expanding field, $\mathscr{L}_{\mathrm{s}}$ is $\mathrm{x}(\partial / \partial \mathrm{x})+\mathrm{y}(\partial / \partial \mathrm{y})$, which is obtained from Equation 3 by setting $f(x, y)=x$ and $g(x, y)=y$; the associated orbits are a star of radial lines. The Lie operator for frontal rotation $\mathscr{L}_{0}$, is $-\mathrm{y}(\partial / \partial \mathrm{x})+$ $\mathbf{x}(\partial / \partial \mathbf{y})$, and it generates a series of concentric circles (bullseye). These two sets of orbits are shown in the second panel of Figure 6 and, as for the first pair, express transformations under which $P R$ is (within reasonable limits) invariant. Notice that the orbits are mutually orthogonal (if superimposed they would always meet at right angles) as are the horizontal and vertical pair. The basic Lie orbits of the primitive theory always occur in such mutually orthogonal pairs (Figure 6; see also section V).

\section{Relations Between Generator and Orbit}

We have talked of trajectories or orbits, which are smooth curves, expressible in simple mathematical equations, and of Lie operators that generate such orbits, operators which in our case also have simple mathematical expressions. What is the relationship 


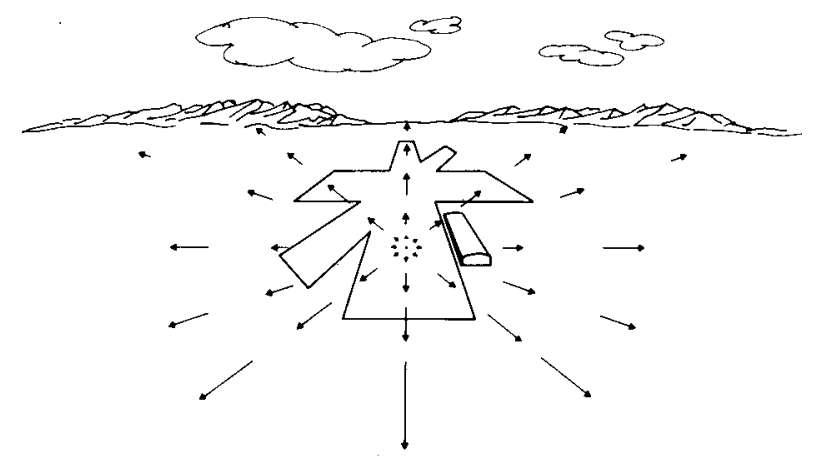

OPTICAL FLOW PATTERN (DILATION) FOR APPROACH

Figure 5. Action of the dilation operator $\mathscr{L}_{\mathrm{s}}$, and its transformation group, in a real-world situntion. The visual field expands from the "straight-ahead" point of aim for landing an aircraft.

between the generators and the orbits they generate? Can one be found from the other? The answer to that second question is yes, but to prove it would take us much beyond the scope of this paper, although a straightforward example is hinted at in Equations 2 and 3 and Figure $3 .{ }^{3}$ The general relationship between any function $F$ that describes a trajectory and the Lie operator $\mathscr{L}^{\prime}$ that generates that trajectory is simple: it is that $\mathscr{L} \mathrm{F} \equiv 0$. In words: if the results of applying operator $\mathscr{L}$ to the function $\mathrm{F}$ is identically zero, then the operator is the one which generates that function. Example: $F_{1}=y-b=0$, which is the equation of a horizontal straight line $(y=b)$. We stated above that $\partial / \partial \mathrm{x}$ is the operator that generates horizontal straight lines. In terms of the relationship just described, then, the application of $\partial / \partial x$ to $F_{1}$ should give an output equal to zero. Operating on $F_{1}$ with $\partial / \partial x$ gives the expression

$$
\frac{\partial}{\partial x}\left(F_{1}\right)=\frac{\partial}{\partial x}(y-b) \equiv 0
$$

(partial differentiation with respect to $x, y$ being considered constant; the result is 0 ). On the other hand, applying $\partial / \partial y$ to $F_{1}$ gives:

$$
\frac{\partial}{\partial y}\left(F_{1}\right)=\frac{\partial}{\partial y}(y-b)=1 .
$$

To take the example of a circle again:

$$
F_{2}=x^{2}+y^{2}-r^{2}=0 .
$$

Operating on this with $\mathscr{L}_{0}$ yields the expression

$$
\begin{aligned}
\mathscr{L}_{0}\left(\mathrm{~F}_{2}\right) & =\left(-\mathrm{y} \frac{\partial}{\partial \mathrm{x}}+\mathrm{x} \frac{\partial}{\partial \mathrm{y}}\right)\left(\mathrm{x}^{2}+\mathrm{y}^{2}-\mathrm{r}^{2}\right) \\
& =-2 \mathrm{yx}+2 \mathrm{xy} \equiv 0
\end{aligned}
$$

and again the output is identically zero (i.e., zero under any conceivable values of $x$ and $y$ ).

This relationship between the operators and their orbits is basic to an understanding of how pattern recognition and Lie group transformations are related. So far I have argued that these are "natural" transformations, and that vision is "transparent" to them. It is not the case that we are unaware of them, but they do not disrupt our perception of the visual

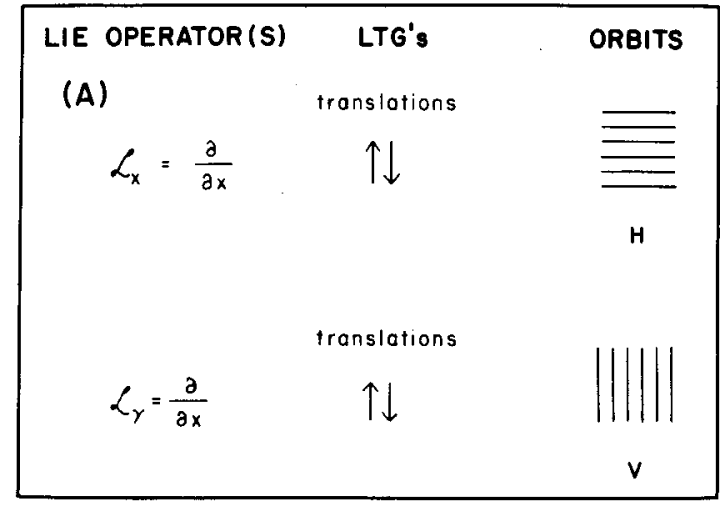
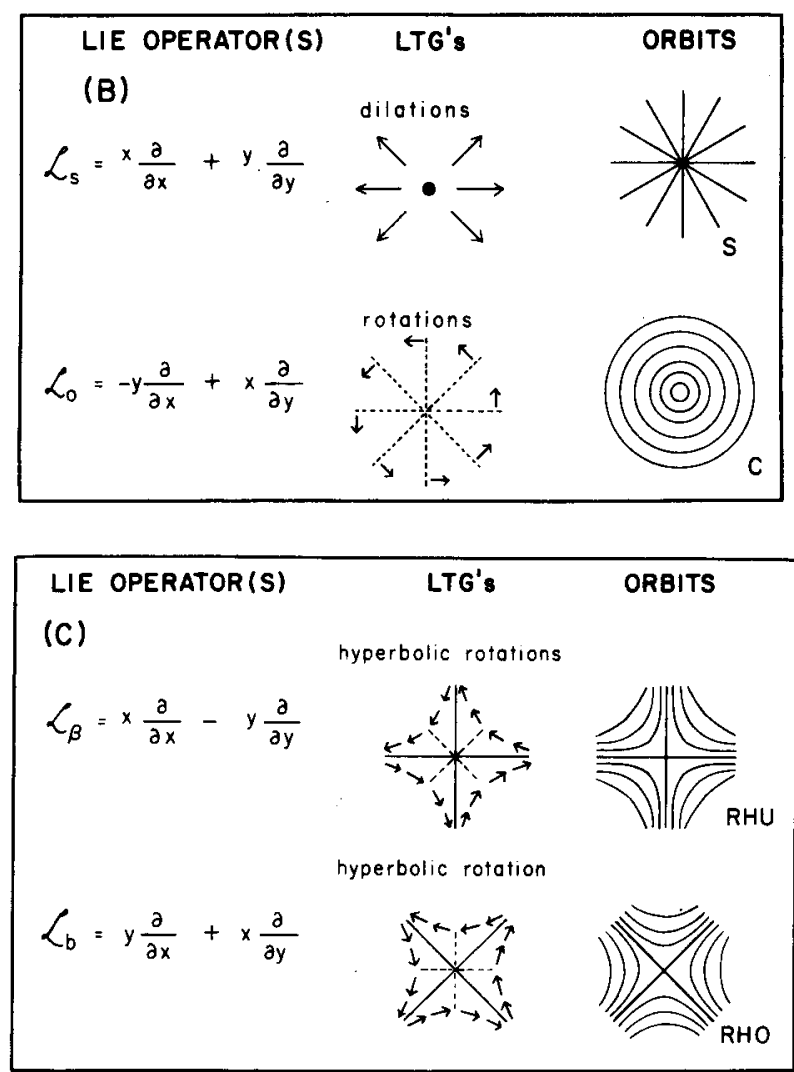

Figure 6. The Lle operators of the primitive Lie theory are shown in three orthogonal pairs on the left-hand side of $A, B$, and $C$, respectively. The transformation groups that they implement and the orbits they generate (on the right) are also illustrated. 
world. In section VI, I will show that the orbits themselves have important properties as patterns.

So far, I have argued that there are certain types of perceptual transformation to which any perceiving organism is subject, because those transformations are rooted in some very fundamental aspects of the organism's relations to its physical environment. It has been shown that the transformations, both at the microgenetic and the macroscopic levels, can be characterized by a particular type of mathematical transformation and transformation group. The next question to ask, obviously, is: Are there other transformations of the same general class which can be applied to visual processing, and which have such a straightforward interpretation in terms of interactions between the perceiving organism and its environment.

\section{LIE ALGEBRA AND PATTERN RECOGNITION}

\section{What Is a Lie Algebra?}

The two sets of orthogonal Lie operators identified above (Figures 6a and 6b), and their associated orbits, are elements in a formal system called a "Lie algebra." In looking for other pairs of orbits that might be relevant in vision, we could approach the question from the ecological point of view, as before, by asking whether there are other "natural" or "ecologically valid" transformations which, because of their importance and ubiquity, could be expected to be ingrained in the visual system's structure. Or we can ask the question: Does the formal system of which the first two pairs are members suggest other operators and orbits? This is a mathematical criterion, but in its own way it is quite compelling, because, under an appropriate rule of combination, it turns out that there is a limited set of pairs of operators. It would take us far afield to go into details here, but the following outline gives the flavor of what is involved.

Lie operators can be combined in various ways; for example, they can be applied serially (one followed by another) or they can be put together in linear combinations; for example, $\alpha(\partial / \partial x)+\beta(\partial / \partial y)$ is a linear combination of the two simple operators $\partial / \partial x$ and $\partial / \partial y$, forming the operator for an oblique translation. In addition, there is a rule for the "composition" of Lie operators called "Commutation" which represents in a certain respect the product of different vectorfield elements. The law of composition is

$$
\left[\mathscr{L}_{\mathrm{i}}, \mathscr{L}_{\mathrm{j}}\right]=\mathscr{L}_{\mathrm{i}} \mathscr{L}_{\mathrm{j}}-\mathscr{L}_{\mathrm{j}} \mathscr{L}_{\mathrm{i}}
$$

In this expression, $\mathscr{L}_{\mathrm{i}}$ and $\mathscr{L}_{\mathrm{j}}$ are any two Lie operators defined as in Equation 3. The new operator $\left[\mathscr{L}_{\mathrm{i}}, \mathscr{L}_{\mathrm{j}}\right.$ ], it turns out, also satisfies the same defini- tion. That is, it too is a Lie operator. If a set of Lie operators has, in addition, the property that the composition of any two of the operators always produces another member of the set, we say that the set-already endowed with the group properties discussed in section II1 - forms a Lie algebra.

The importance of this conception for us is twofold: first, the number of possible operators in the system is fixed and finite (because, by commuting pairs of them, one comes back to another member of the original group of operators). Second, it suggests that one should try to establish whether or not such algebraic relationships have a plausible visual interpretation. If so, it means that there is a delimited class of vectorfield structures and their associated orbits that define the basic properties of visual space and object transformations within it. That is to say, it should be the case that orbits generated by other members of this group, and particularly by the simplest of those member pairs, should have some clear representation in visual patterns and should share some properties with the two pairs of orbits already discussed.

\section{The Third Orbit Pair}

The next pair of operators to consider generate sets of rectangular hyperbolas, and these form the third fundamental pair of the primitive theory. In the first instance, their inclusion was suggested by the algebraic structure indicated by the nature of the first two pairs; $\mathscr{L}_{\mathrm{x}}, \mathscr{L}_{\mathrm{y}}$ and $\mathscr{L}_{0}, \mathscr{L}_{\mathrm{s}}$. The operators and their orbits are shown as panel $\mathrm{C}$ of Figure 6. What is their interpretation in terms of visual processing? Hoffman identifies the operators with properties of binocular space which, in its simple and general form, is hyperbolic (Luneburg, 1946; Indow, 1979). Although the interpretation is a little less obvious than for the first two pairs, one can, nevertheless, consider these rectangular hyperbolas as the plane projections of hyperbolic orbits in binocular space (Hoffman, 1966). As will be discussed later, this orbit pair does share some of the properties of the first two pairs, and so can be considered to be a reasonable candidate for inclusion in a model of visual processing.

\section{Extension to Other Operators and Orbits}

How much further can one take this process of choosing operators by a mathematical criterion, and then of looking for a perceptual interpretation? The answer is quite far, but we shall not pursue the matter. To indicate where it can lead, however, it is obvious that another dimension that enters in a very fundamental way into visual perception is time. If we allow operations in time, then the relevant differential operator is $\partial / \partial t$.

We can reach a high level of generality by considering all the simple operators that might function 
in "plane-time," which, in mathematical language, is described as $R^{2} \times T$. The so-called general linear group for $R^{2} \times T$ is

$\begin{array}{llll}\partial x & x \partial x & y \partial x & t \partial x \\ \partial y & x \partial y & y \partial y & t \partial y \\ \partial t & x \partial t & y \partial t & t \partial t\end{array}$

(where $\partial \mathrm{x}$ stands for $\partial / \partial \mathrm{x}$, etc.).

Only certain subgroups of these operators and their combinations appear to be appropriate to perception, but these subgroups can be shown to meet the criterion for a Lie algebra. Figure 7, which is a re-

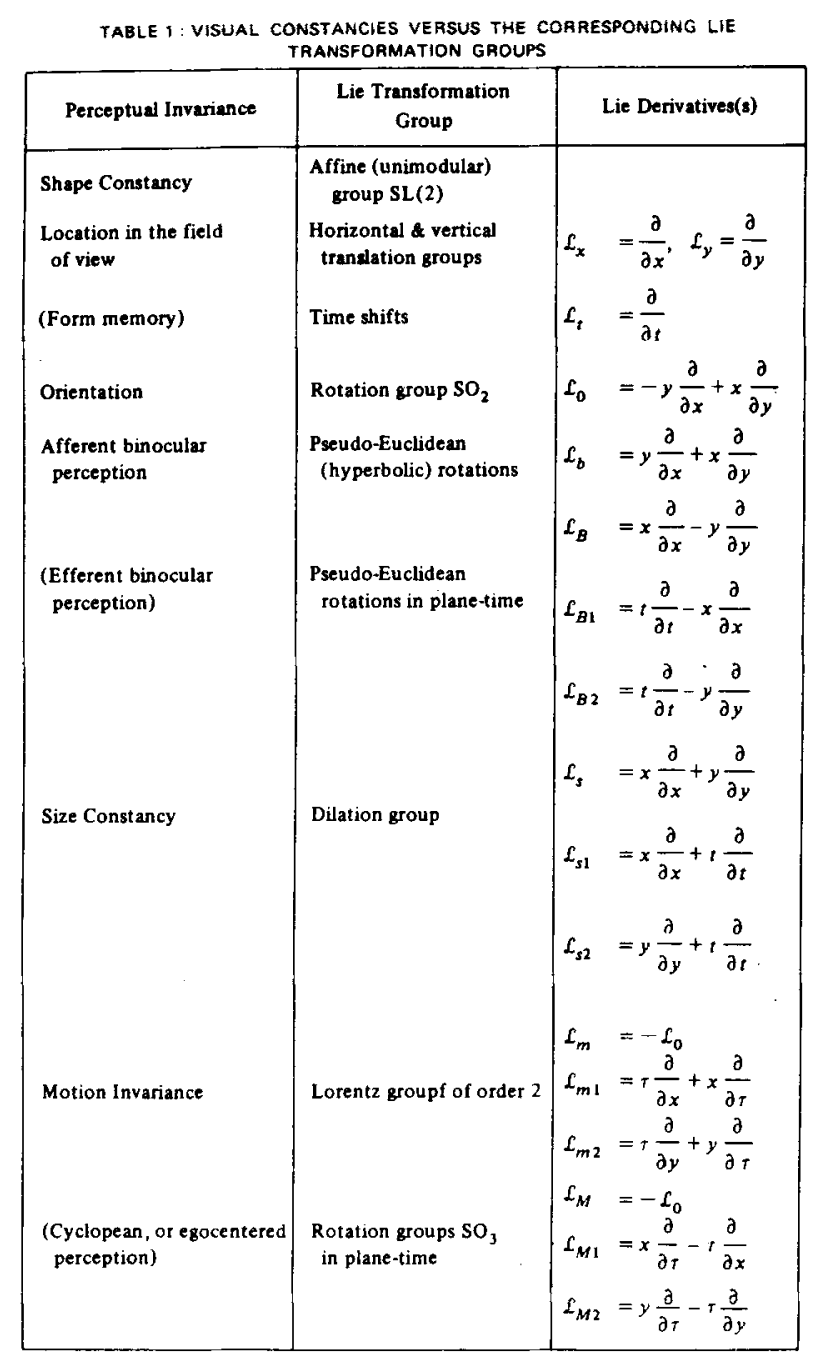

$x=$ horizontal distance trom the perceptual center on whatever frame of reference is appitcable : $y=$ corresponding vertical distance $: \tau=c^{\prime} r$.

$t=$ tame measured from the Ohserver's present in cortical (neuropsychologacal) units, $c^{\prime}=$ maximum flow velocity of cortical signals.

Figure 7. Hoffman's table of the basic constancies and the corresponding Lie transformation group and perceptual invariance (from Hoffman, 1977). production of Table 1 from Hoffman (1977), indicates some of the fundamental Lie derivatives and their relationship to what Hoffman calls the constancies. It should be clear that what Hoffman called a constancy has here been related to invariance under transformation in the Gibsonian sense. The full multiplication table for the Lie algebra of mature visual perception (Hoffman, 1977) consists of 17 "elementary" operators similar in form to those shown in Table 1 , together with their products $\left[\mathscr{L}_{\mathrm{i}}, \mathscr{L}_{\mathrm{j}}\right]$. Of course, the Lie products themselves turn out to be members of the original set, because of the group property discussed earlier.

This digression serves to indicate the scope of even the elementary form of the theory. The important point is that, from considering the general mathematical structure of LTGs, one can pick out subsets which are relevant to perception, and which themselves form a group in the technical sense.

\section{PATTERN RECOGNITION}

\section{The Recognition of Simple Patterns: \\ "Matching" or "Cancellation"}

It should now be clear that a major postulate of the LTG theory is that the basic structure of the visual manifold is determined by the simple Lie operators that express its vectorfield components, and that these, in turn, are at least to a high degree determined by the ecological and evolutionary constraints under which visual systems have developed. What I have called the "primitive model" (not Hoffman's name for it) asserts that the three-orbit structures of $\mathscr{L}_{x}$, $\mathscr{L}_{\mathrm{y}}, \mathscr{L}_{0}, \mathscr{L}_{\mathrm{s}}$, and $\mathscr{L}_{\mathrm{b}}, \mathscr{L}_{\mathrm{B}}$ are embedded in the visual manifold. I have singled these out just because they are easy to understand and visualize, and secondly because if it (the primitive model) should turn out to be wrong, LTG/NP would lose much of its plausibility (see section VIII1 below).

Having approached the question of vectorfield structure from the point of view of transformations and their computation, it is now necessary to introduce the problem of pattern recognition, and how this is dealt with in LTG/NP. The first point to make is that in LTG/NP recognition of a pattern occurs by a process of cancellation; to put it more specifically, the visual system seeks those differential operators which will reduce the output of any path (i.e., a visual contour) to zero. This is an implementation of the rule $\mathscr{L} \mathrm{F}=0$ (section IV3). In the first instance, the operators of the primitive model are applied; if the resultant output (for one of them) is zero, then the relevant orbit is recognized. Effectively, the system is set to "resonate" to the simplest structures. Thus, we have a new definition of what constitutes a "simple" pattern. If the path output is nonzeroas will certainly be true in the vast majority of naturally occurring situations-other "higher" forms of operator are invoked, as I shall indicate directly. 
This process of attempted recognition occurs "automatically" for every input pattern imposed on the visual manifold, and is not to be confused with the computation of transformations over time. When the simple operators are applied over time to more complex patterns, the result will be transformations across the manifold (translations, dilations, rotations), as previously explained.

\section{Recognition of Complex Patterns: Prolongations and Differential Invariance}

The path curves of most patterns are not simple orbits of the primitive Lie operators. To be of use as a general theory of visual perception, it must be possible to explain in similar terms the recognition of more complex patterns. This can be done, and I shall attempt to give an outline of the extended model. However, to expound it fully would go far beyond the treatment that is appropriate here. The explanation of the recognition of more complex patterns is attained through the notion of the "prolongation" of simple operators to higher order differential operators, and of higher order differential invariance.

We call Expression 3 a simple operator because it involves only first-order differential terms. An expression like $\mathrm{d} / \mathrm{dx}$ is a first-order operator because it is the instruction to differentiate with respect to $x$. The expression

$$
\frac{d}{d x}\left(\frac{d}{d x}\right)=\frac{d^{2}}{d x^{2}}
$$

is a second-order operator, because it is the instruction to differentiate twice with respect to $x$. Similarly, other differential operators can be of second or higher order, depending on the number of successive differentiations involved. These same ideas are true also with respect to partial differentiation, the expressions being $\partial^{2} / \partial x^{2}, \partial^{2} / \partial y^{2}$ and $\partial^{2} / \partial x \partial y, \partial^{2} /$ $\partial y \partial x$, etc. Just as an expression like $d y / d x$ can be understood as an expression for the rate at which $y$ is changing with respect to $x$, so the expression $d^{2} x / d y^{2}$ can be thought of as the rate at which the rate of change of $y$ with respect to $x$ is itself changing (this will be familiar to many as an expression for acceleration). Although we did not prove it, it seems intuitively clear that the differential operators of the first order, containing only simple expressions involving $\partial / \partial x$ and $\partial / \partial y$ generate smooth and rather simple curves, and it can be guessed that an operator involving higher order expressions will generate a more complex curve. Conversely, we can say that a complex curve (in our case, a more complicated visual contour) will have as its "matching" or "canceling" operator something which involves more differential terms than the first-order simple $\partial / \partial x$ and $\partial / \partial y$. An important insight is Hoffman's conjecture that these are not just arbitrary complex op- erators, but are the so-called "prolongations" of the basic operators (Hoffman, 1970). Thus, as I shall describe more fully below, even complex forms are in an important sense related to-even derived fromthe basic orbits of the primitive model.

Theoretically, a curve of any degree of complexity can be "matched" by a differential operator of sufficiently high order. A proof of this statement would be much beyond us, but the general idea is not so hard to grasp. It is rather like the situation in two-dimensional analytical geometry, where it can be shown that a curve through $\mathbf{n}$ points in the plane can be fully determined with $\mathrm{n}$ constants. This means that the more points there are, the more complicated the relevant equation will be (including terms in higher powers of $x$ and $y$ and their products), and so the more complicated the curve becomes. Readers familiar with calculus will recognize that the higher the order of such an equation, the more often it will have to be differentiated to get rid of its arbitrary constants. The result is a higher order differential equation that expresses the "essence" of the form, just as our simple differential equation

$$
\frac{d y}{d x}=\frac{-x}{y}
$$

expresses the "essence" of the rotational vectorfield whose orbits are the circles $x^{2}+y^{2}=r^{2}$ (section II3). To quote Hoffman (1970, p. 439): "In addition to the spatial coordinates themselves, higher and higher derivatives must be brought in in order to characterize more and more complicated orbits, that is, visual forms. This ... leads to the concept of differential invariants (Guggenheimer, 1963). Differential invariants are ones that depend not only upon position in the (visual) manifold but also on the derivatives of arbitrarily high order at that point."

Any arbitrarily complex pattern can be "fitted" with an arbitrarily complex operator. However, it is important to remember that the complexities are, in a sense, built upon an original set consisting of relatively few fundamental operators: One may conjecture that the visual system, in trying to "match" an arbitrarily complex curve, will attempt to do so with the lowest order of differential operators possible, and hence strive for the simplicity of the "good figures" of Gestalt psychology (Hoffman \& Dodwell, Note 1).

An example of a slightly more complex path and its generator is a periodic curve such as the sine function. This function can be considered to be generated by a simple harmonic motion in the vertical dimension and a constant motion in the horizontal dimension (each measured in terms of some "independent parameter," say t). The operator for the latter is, of course, $\mathscr{L}_{\mathrm{x}}=\partial / \partial \mathrm{x}$, as we have seen earlier; the operator for the simple harmonic motion depends 
on the fact that this motion involves an accelerative component, so we will expect to find a second-order differential term in it. In fact, the operators are $\mathscr{L}_{x}$, and $\mathscr{L}_{1}^{(2)}$, respectively, the latter being the so-called "second prolongation" of the simple operator $\mathscr{L}_{1}=$ $y(\partial / \partial y) .{ }^{4}$ Thus, we see that one step up in complication involves us already in a second-degree operator; the principle is the same for any arbitrarily high degree of complexity.

It will be immediately apparent that the LTG/NP model is very powerful; in fact, it can account for the recognition of any pattern whatsoever, although there will, of course, be differences in the "ease of recognition." All one needs to do is to postulate a sufficiently high level of differential invariance to "cancel" or "annul" any pattern. That being so, one might ask what the testable consequences of the model might be. On the assumption that ease of discrimination is related to the degree of the operators needed to cancel or recognize a pattern, it clearly does have some testable consequences. As it has been outlined here, the primitive model predicts that patterns involving only its operators and orbits should be fundamental in vision, and be the easiest to discriminate. One might, for example, expect such patterns to be "simple" for the young organism to learn, and to be particularly salient for the adult organism. Most of the tests derived so far have, in fact, addressed questions of this sort. See section VIIII below.

\section{LTG/NP AS A THEORY OF VISUAL PERCEPTION}

\section{Character of the General Model}

I have presented LTG/NP in a manner somewhat different from Hoffman's, but hope that I have not done violence to its basic concepts. Although Hoffman talks of the constancies and the mathematical invariances that underlie them, it has become increasingly clear to me that most psychologists will find an approach via the Gibsonian notion of transformation and invariance under transformation more congenial. I like to think that LTG/NP actually complements the Gibsonian approach in a very direct way, by telling us what the general principles of computation underlying transformations must be, and giving clear mathematical expression to their formal character. In this sense, it supplies a geometry (differential geometry) of a type which Gibson often claimed to be seeking, but evidently never found. This failure of the Gibsonian school to take advantage of the formalism supplied by LTG/NP has been remarked on-and lamented-before (Zusne, 1970).

The LTG/NP explanation of the simple transformations and the orbits they imply is pretty straightforward, and in a sense has to be true. That is to say, there is no doubt that the transformations we have discussed occur continually in a normal visual organism, and also little doubt that under those transformations the perceptual world remains stable. It is easy enough to see that the same types of continuous transformation must apply in the time domain. In this sense, the primitive model of LTG/NP is akin to Luneburg's theory of binocular visual space. Luneburg (1947) was concerned with solving the problem of a coherent one-to-one mapping between points in physical space and their representation via the "bipolar" visual code of two eyes, each with its own characteristic retinal signature. He did so by specifying the geometry of a system that implements such a mapping. Even if wrong in detail, Luneburg's model, or something closely similar to it, has to be correct, given the evident fact of useful stereoscopic binocular vision (Dodwell, 1982). In the same vein, Hoffman's model specifies processes for mapping characteristics of the physical world into a dynamic visual system. This model, or one along closely similar lines, has to be correct, given the evident fact of stable spatial vision and pattern recognition under the vagaries of the perceiver's locomotion. In both cases, the mathematical formalism involved lends a striking force and clarity to the conception.

\section{Beyond the Primitive Model}

A major point of interest, and one which should engage our attention and discussion, is the question of how far one can go beyond the simple orbits of the primitive model. There are two questions to raise. The first is: can one attribute ecological or evolutionary significance to any transformation other than the simple ones we have described? Hoffman argues that one can (Hoffman, 1966, 1970, 1977), but many psychologists will find his arguments not completely convincing. Even in the case of the hyperbolic orbits of the third transformation pair we have discussed, it is not totally obvious that the orbits play a necessary role in the normal perceptual environment. It will require a good deal more thought and experimentation to convince many psychologists that the more complex orbits and their transformations really do describe cogent factors in normal visual processing. The second major question concerns the recognition of more complex patterns. The question of how this is to be separated from the generation of the transformation groups themselves has not always been clearly enunciated. As soon as one goes beyond the simple transformations of the primitive model, there is a real question of the extent to which transformation groups and orbits have the dual characteristic (transform generator and visual contour) we have emphasized. Although the higher differential invariants needed for the recognition of complex patterns, such as a face, are derived from the basic orbits (via prolongation: section VI2), they do not so easily "fall into place" as natural conse- 
quences of the ecological desideratum of a stable perceptual environment. On the other hand, the elegance of the mathematical solution has strong appeal. It specifies just those local characteristics that are sufficient for pattern recognition to occur. Notice, however, that the characteristics are local with respect to the general pattern configuration, not in the restrictive sense of coding only for a circumscribed area of the visual field, or retina. Thus, the differential invariants are elaborations on whatever structure a pattern may have in terms of concatenations of segments of the basic Lie orbits (cf. Hoffman \& Dodwell, Note 1); they do not exist "in vacuo." The operation of recognition, however, is still one of matching, or cancellation, of the visual contours by whatever level of operator may be required.

While this aspect of the model is powerful and elegant, one can still ask the question: Is it a true account of visual recognition?

\section{Testability of the General Model}

There are real problems with testing the validity of the general model experimentally. As I remarked earlier, the theory is so powerful that it can be said to be compatible with any state of affairs so far as the discrimination of different patterns is concerned. However, it should be possible to test the notion of ease of discrimination and simplicity of the operators by looking for correlations between complexity in the Lie description of a pattern and its recognizability, and a beginning on this work has indeed been made (see section VIII1 below).

The question of testability actually involves many factors, even in terms of the primitive theory. One problem is that any smooth curve can be approximated over some region by a Lie orbit. For example, any reasonably small portion of a parabola can be approximated by a segment of a circle, a hyperbola, or a straight line. It is not absolutely certain even that the simple Lie orbits of the primitive model are fundamental. It could be that any smooth curve has properties of redundancy and "good form" which make it a candidate for simple processing, just as such a smooth curve is likely generated by a Lie operator, albeit not one from our special set. The question of approximation to a Lie orbit is a serious one, particularly as we do not presently know what "local" means in the field where the Lie operators may be functioning, or what degree of approximation the visual system tolerates. But such questions should be amenable to experimental test.

\section{Conditions for Integration of \\ Locally Defined Codes}

The idea that visual forms can be "measured" by receptive field units of the Hubel and Wiesel type has been around in psychology for quite some time (e.g., Dodwell, 1970, 1978; Marr, 1976). It is recog- nized by these and other authors that the major theoretical problem is to model the powerful integrative action of the visual system. The LTG/NP model of how both the measurement and representation of local contours is achieved, and of how integration occurs, is far more profound than other models that have been proposed, if only because it addresses the question of how such measurement and integration can lead to coherence in the processed image. In approaching the problem of coherence from the point of view of vectorfield operations, we find there is an important theorem that proves what the relations between the local operators have to be in order to yield orbits or trajectories, which in LTG/NP are, of course, visual contours. What is required is that the local vectors can be lined up head to tail in the manner suggested in Figure $2 b$, rather than being scattered across the field in an incoherent fashion. A vectorfield which has this property of holonomy of the local vectors, is known as a "contact structure." The theorem of Frobenius states that a necessary and sufficient condition for holonomy to hold is that locally the vectorfield must have a Lie group structure. Without going into any of the mathematical statement, this means that for vectorfield operations to work at all in vision, it has to be the case that the structure is like the one we have described. Recall the dual character of the processing postulated in LTG/NP. Vectors specify the local (linearly approximating) fit to imposed stimulation, in this sense being a sort of minimal local template, whereas the vectorfield specifies global properties (orbits, visual contours). The theorem of Frobenius sets the mathematical condition for this to be possible. I argued that this structure could be understood as arising from the ecological and evolutionary constraints under which a visual organism develops, so we have the remarkable fact that it cannot be surprising that LTGs are at the basis of pattern recognition; it has to be the case, given the constraints of the natural world within which visual systems develop. This idea is further elaborated in a recent paper by Hoffman and Dodwell (Note 1).

One can summarize this discussion by saying, first, that if vectorfield operations are relevant in vision, they must generate smooth trajectories which are interpreted as visual contours; second, if that happens, that the local structure of the vectorfield must conform to the conditions stated in the theorem of Frobenius; and third, that this, in turn, means that the local structure is that of the Lie derivatives and the transformation groups that they imply.

\section{Local Contour Generators \\ and Cellular Morphology}

Hoffman (1968) has identified the so-called "Lie germs," or the local processes that operate as the Lie derivatives, with various sorts of cell morphology 
in the visual cortex. For instance, he compares Sholl's (1956) seven principle cortical cell types with the functions they might perform as local "graphical integrators" in forming visual contours. Most recently, he has extended this form of analysis to consideration of how certain empirically determined models of cortical circuitry (Bishop, Coombs, \& Henry, 1971; Shepherd, 1974) might implement the integrations required by LTG/NP (Hoffman, 1983). This aspect of the theory is somewhat speculative, and it is important to realize that the application of the mathematics in general to visual processes does not rest logically upon it. I make the point explicitly because some critics have held that LTG/NP is worthless unless the identification of the different cell types' functions could be shown to be correct. Such an identification would undoubtedly add powerful support to the model, if proven by physiological methods, but its plausibility does not rest solely on that point.

To my mind, there is still something of a gap between the general postulation of cortical cells as functional elements of a vectorfield (section III1) and the more detailed speculation about cell morphology, the nature of local neuronal flows, and their action in cortical network models. The former aspect of cell function is well established in the neurophysiological literature on single-cell recording in the visual system; the latter is not. The idea that cells of different morphological types have different functions is plausible enough, but there is as yet no evidence at the functional neurophysiological level (e.g., singleunit recording) to support the notion of different orbital integrative action for them. This aspect of the theory is certainly controversial, and it seems that with the rapid growth in understanding of the relation between cellular form and function in contemporary neuroscience, it should be possible to test Hoffman's conjectures in the relatively near future. As that aspect of the theory is not central to understanding the general mathematical structure, I have not discussed it in detail.

\section{APPRAISAL OF LTG/NP}

\section{Empirical Support}

LTG/NP was not conceived "in vacuo"; at the time of its inception, a large amount of the literature of the experimental study of vision was held to be consistent with it, and specific findings such as MacKay's (1961) complementary after images and Bhatia and Verghese's (1964) demonstration of threshold changes in length with speed, as well as constancy phenomena in general, were quoted as support for the model (Hoffman, 1966).

The first experimental studies designed to test particular predictions derived from LTG/NP were conducted by Caelli (1974). Since then, a number of re- ports have appeared, or will shortly do so. The experiments are of four sorts: (a) those that test spatiotemporal predictions from the theory; (b) those that test pattern interactions predicted by the theory, including illusions; (c) those that test "saliency" or ease-of-discrimination of Lie patterns, and (d) those that examine adaptational phenomena related specifically to Lie patterns.

(a) In two papers, Caelli, Hoffman, and Lindman (1978a, 1978b) describe experiments on relativistic effects in real and apparent motion perception that are directly predicted from the Lorentz transformations of special relativity. These, in turn, are derived from the Lie orbits in space-time, because the infinitesimal generators of the Lorentz transformation group are Lie operators, as defined earlier in section II1. These experiments represent a quantum leap forward in understanding and the quantitative modeling of visual motion phenomena. Experiments by Foster (1975, 1978) also demonstrate the importance of Lie orbits in the prediction of the paths of apparent motion (see also Caelli \& Dodwell, 1980).

(b) Caelli $(1976,1977)$ reports experiments on the recognition of global forms (e.g., a circle) as a function of the alignment of local tangent vectors, and on the detection of such patterns in visual noise. Of particular interest here is the question of commutability (section V1); he was able to show that thresholds for the detection of global patterns formed by the conjunction of orthogonal and commuting operators are lower than for nonorthogonal and noncommuting pairs. These are the only experiments so far to address specifically the algebraic properties of LTG/NP. Some results with illusory figures, which also support the model, have been reported by Caelli, Finlay, and Hall (1975) and Smith (1975), and discussed by Hoffman (Note 2).

(c) Dodwell, Wilkinson, and von Grunau (in press) and Wilkinson and Dodwell (1980) have examined the prediction that patterns made up of the simple Lie orbits (see Figure 6) should be salient and easy to discriminate for young organisms. In a series of experiments with kittens, this prediction was supported; we were also able to show that, in general, Lie patterns are easier for the kitten to discriminate than are non-Lie patterns of similar manifest complexity. Dodwell, Humphrey, and Muir (1983) have shown that babies at 4 months of age also respond to the organizational properties of a "fragmented" Lie pattern-what we now call a vectorgraph. Caelli and Dodwell (1982) have studied vectorgraph recognition; a vectorgraph is a two-dimensional pattern made up of short line segments, as illustrated in Figure 8. These can represent a vectorfield more or less fully, as each element has both a defined position and orientation (compare with Figure 3). We found that ease of discrimination, and the fineness of "tuning" of local position and orientation codes, is strongly 


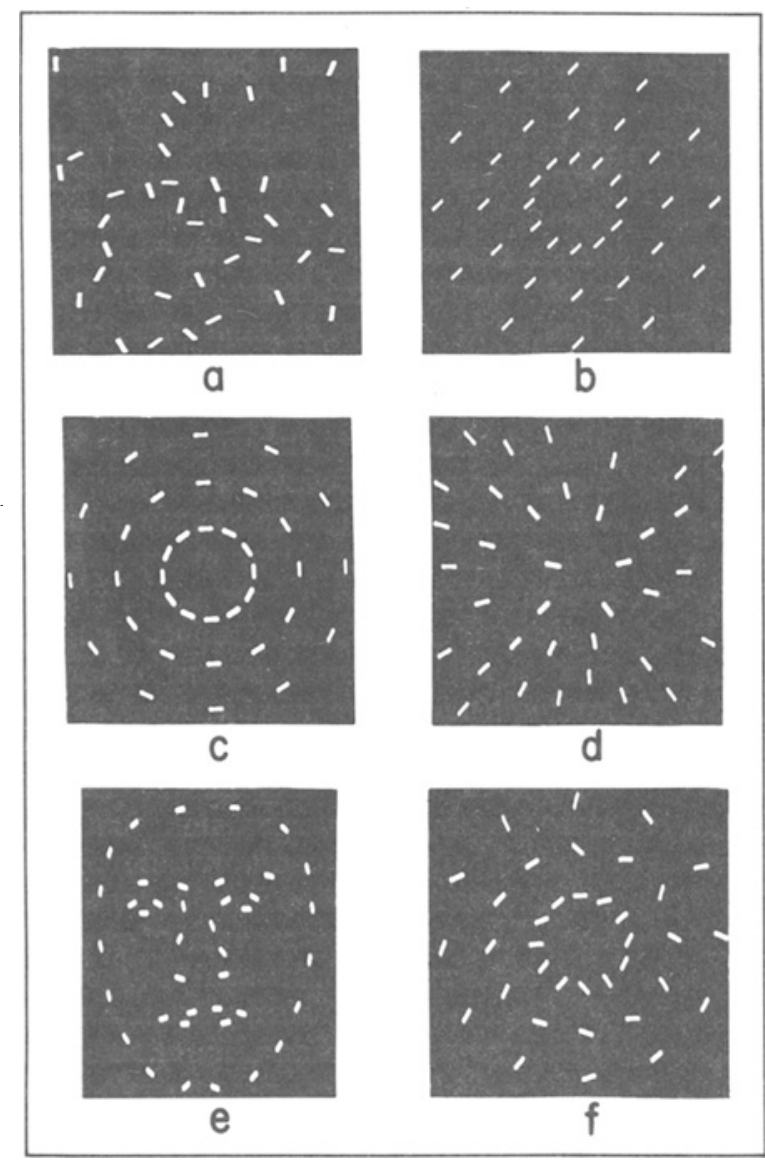

Figure 8. Examples of vectorgraphs, which are partial representations of vectorfields specified by position and orientation codes (compare with Figure 3). The vectorgraphs ahown vary from one with random position and orientation codes (a), through figures embodying some of the one-parameter transformation groups (c and d), to a "vectorsketch" of a face (e).

affected by (global) vectorfield structure, and that the vectorfields of one-parameter transformation groups such as translation, dilation, and rotation (in effect, vectorfields of the Lie orbits of the primitive model) are especially powerful in this regard. In a second study (Dodwell \& Caelli, Note 3), we have shown that the effects on local coding stand up, essentially, even under an imposed transformation of the vectorgraph such as rotation.

(d) Using a McCollough-effect paradigm, we have shown (Dodwell, Note 4; Emerson, Humphrey, \& Dodwell, Note 5) that the Lie patterns of Figure 6 can be used to induce whole-field pattern-contingent color aftereffects, and that three independent aftereffects, generated by the three orbit pairs of the primitive model, can be demonstrated simultaneously in the same subject. These effects are found only when the color-pattern combinations are those appropriate to the theoretically related pairs of patterns. This is the first demonstration that the three Lie pairs of the primitive model may be three independent pattern- generating "channels," on the basis of which further shape recognition operations are elaborated, as described earlier in section VI2.

Thus, we see that, although the experimental work has been confined mainly to a few laboratories and investigators (the list is not exhaustive of all relevant work, incidentally), there is now considerable new support for LTG/NP. While no one of these experiments is in itself a critical test of the model, in the sense that no other interpretation of the outcome is possible, their cumulative effect is to strengthen the claims of the model as a serious candidate for the status of an overall structure within which processes of pattern recognition can be described and understood.

\section{Theoretical Status}

First, I must emphasize that LTG/NP, as described here, is very much a model of what I have elsewhere termed "level 2" processing (Dodwell, 1978). It is a level that goes beyond the consideration of merely local processes that might, for instance, be embodied in the actions of single neurons. It deals with organizational and transformational (field-like) effects in vision, but at a level short of what we can identify as full cognitive functioning. While the LTG/NP model described here is compatible with a more general mathematical theory of cognitive functioning (Hoffman, 1980), it is not dependent on that theory; its validity is to be assessed independently of that theory.

It is surprising, or at least I am surprised, that the LTG/NP has had so little influence in psychology, because, when measured against a reasonable set of criteria for a neuropsychological theory, ${ }^{3}$ it comes out rather well (Dodwell, 1977). In evaluating the theory from a very general point of view (Dodwell, 1977), I followed Mario Bunge's (1963) work on the evaluation of scientific theories to a great extent. Any reasonable neuropsychological theory of perception would need to account for many of the phenomena in visual perception, and the theory would have to have some depth. That is to say, it should be able to relate different aspects of visual processing to each other. This LTG/NP certainly does, in giving us a very explicit account of how one can go from the local feature detector to more global operations. In fact, it does a more adequate job of this than any other I can think of. It also bridges the gap between the two cornerstones of spatial vision, pattern and object perception-principles of invariance, on the one hand, and processes of local coding or filtering, on the other. A model must have predictive power, and LTG/NP certainly has this. We have discussed the difficulties of testing its more complex aspects, but certainly in its simple form it yields testable predictions. Then, a theory must have extensibility. However precise the original formulation of a model may be, it must be possible to show that it has wide ap- 
plication over the field of enquiry in question. There is little doubt that on this score, too, LTG/NP comes out rather well. A model must also show originality, and one need spend little time in arguing that for LTG/NP. Clearly, in bringing to bear a powerful and original mathematical theory, it constitutes an original contribution to visual perception. A model should also be parsimonious, provided that parsimony is not bought at the expense of richness of application. LTG/NP is parsimonious in the sense that it develops from a few rather precise ideas about the genesis of perceptual events, and, despite this, it has a very rich field of application. Finally, a theory to be taken seriously must have "world view compatibility." LTG/NP sits very comfortably with our notion that there are neural processes underlying the perception of visual contours and patterns, and from that point of view does not strain our credulity.

LTG/NP meets the criteria reasonably well. It has had little impact, first because the theory is difficult, and second, because it was not couched in language that is familiar to psychologists, or at least not in language that was obviously compatible with the mainlines of psychological theorizing in the field of vision. I have aimed to do two things in this paper: first, to make the mathematical ideas, and hence the model, more accessible to psychologists, and second, to show that the theory actually does fall right in the mainstream of current thinking on the importance of transformations in perception, and also the renewed interest in the prominence of Gestalt-like factors, which transcend the detection of local features. I believe it is generally true that inability to forge a convincing theoretical path from the local to the global is a weakness of most psychological theorizing about perception (Dodwell, 1982); it is a weakness which I have tried to show can be overcome by the LTG/NP model as a theory of how visual contours are detected, represented, and integrated. Vectors and vectorfields surely have an obvious relevance in vision; what we have lacked heretofore is a general model of their action and a theory of how that model is embodied in a real visual system and real visual activity.

\section{REFERENCE NOTES}

1. Hoffman, W. C., \& Dodwell, P. C. Geometric psychology generates the visual Gestalt. Manuscript submitted for publication, 1982.

2. Hoffman, W. C. In defense of LTG/NP (Monograph). Marseilles: Institut de Neurophysiologie et Psychophysiologie, CNRS, 1976.

3. Dodwell, P. C., \& Caelli, T. M. Recognition of vectorgraphs under transformations: Local and global determinants. Manuscript submitted for publication, 1982.

4. Dodwell, P. C. The geometry of vision. Address at L.O.V.E. Conference, Niagara Falls, Ontario, March 1981.

5. Emerson, V. F., Humphrey, G. K., \& Dodwell, P. C. Contingent color aftereffects: Evidence for an integrative mechanism. Manuscript submitted for publication, 1982.

\section{REFERENCES}

Bhatia, B., \& Verghese, C. A. Threshold size of a moving object as a function of its speed. Journal of the Optical Society of America, 1964, 54, 948-950.

Bishop, P. O., Coombs, J. S., \& Henry, G. H. Interaction effects of visual contours in the discharge frequency of simple striate neurons. Journal of Physiology, 1971, 219, 659-687.

Bruter, C. P. Invitation a la géometrie differentielle. Cahiers de Psychologie, 1977, 20, 73-90.

Bunge, M. The myth of simplicity: Problems of scientific philosophy. Englewood Cliffs, N.J: Prentice-Hall, 1963.

CAELLI, T. M. Models, Lie algebras, and visual pattern perception. Doctoral thesis, University of Newcastle, N.S.W., Australia, 1974.

CAELLI, T. M. The prediction of interactions between visual forms by products of Lie operators. Mathematical Biosciences, 1976, 30, 191-204.

CaElli, T. M. Psychophysical interpretations and experimental evidence for the LTG/NP theory of perception. Cahiers de Psychologie, 1977, 20, 107-134.

CaElli, T. M., \& Dodwell, P. C. On the contours of apparent motion: A new perspective on visual space-time. Biological Cybernetics, 1980, 39, 27-35.

CaElli, T. M., \& Dodwel., P. C. The discrimination of structure in vectorgraphs: Local and global effects. Perception \& Psychophysics, 1982, 32, 314-326.

Caelli, T. M., Finlay, D. C., \& Hall, A. C. Lie transformation groups and the Poggendorf illusion. Australian Journal of Psychology, 1975, 27, 177-186.

Caelli, T. M., Hoffman, W. C., \& Lindman, H. Subjective Lorentz transformations and the perception of motion. Journal of the Optical Society of America, 1978, 68, 402-411. (a)

Caelli, T. M., Hoffman, W. C., \& Lindman, H. Apparent motion: Self-excited oscillations induced by retarded neuronal flows. In E.L.J. Leuwenberg \& H.F.J.M. Buffart (Eds.), Formal theories of visual perception. New York: Wiley, 1978. (b)

Campgell, N. R. Physics: The elements. Cambridge: Cambridge University Press, 1920.

Dodweld, P. C. Visual pattern recognition. New York: Holt Rinehart \& Winston, 1970.

Dodwell, P. C. Criteria for a neuropsychological theory of perception. Cahiers de Psychologie, 1977, 20, 175-182.

DoDWELL, P. C. Human pattern and object perception. In R. Held, H. W. Leibowitz, \& H.-L. Teuber (Eds.), Handbook of sensory physiology. New York: Springer, 1978.

DoDWELL, P. C. Geometrical approaches to visual processing. In D. Ingle, M. Goodale, \& R. Mansfield (Eds.), Analysis of visual behavior. Cambridge, Mass: M.I.T. Press, 1982.

Donwell, P. C., Humphrey, G. K., \& Muir, D. W. Pattern and object perception. In L. Cohen \& P. Salapatek (Eds.), Handbook of infant perception. New York: Academic Press, 1983.

Dodwell, P. C., Wilkinson, F. E., \& von Grunau, M. Pattern recognition in kittens: Performance on Lie patterns. Perception, in press.

Foste R, D. H. Visual apparent motion and some preferred paths in the rotation group $\mathrm{SO}(3)$. Biological Cybernetics, 1975, 18, 81-89.

Foster, D. H. Visual apparent motion and the calculus of variations. In E.L.J. Leeuwenberg \& H.F.J.M. Buffart (Eds.), Formal theories of visual perception. New York: Wiley, 1978.

GiBson, J. J. The perception of the visual world. Boston: Houghton-Mifflin, 1950.

Guggenheimer, H. W. Differential geometry. New York: McGraw-Hill, 1963.

Hofman, W. C. The Lie algebra of visual perception. Journal of Mathematical Psychology, 1966, 3, 65-98; errata, Journal of Mathematical Psychology, 1967, 4, 348-349.

Hoffman, W. C. The neuron as a Lie group germ and a Lie prod- 
uct. Quarterly Journal of Applied Mathematics, 1968, 25, 423-441.

Hoffman, W. C. Higher visual perception as prolongations of the basic Lie transformation group. Mathematical Biosciences, $1970,6,437-471$.

Horfman, W. C. An informal, historical description (with bibliography) of the "L.T.G./N.P." Cahiers de Psychologie, 1977, 20, 139-150.

Hofrman, W. C. Subjective geometry and geometric psychology. Mathematical Modelling, 1980, 1, 349-367.

Hofrman, W. C. Figural synthesis by vectorfields: Geometric neuropsychology. In P. C. Dodwell \& T. M. Caelli (Eds.), Figural synthesis. Hillsdale, N.J: Erlbaum, 1984.

Hubel, D. H., \& Wiesel, T. N. Receptive fields, binocular interaction, and functional architecture in the cat's visual cortex. Journal of Physiology, 1962, 160, 106-154.

HuBeL, D. H., \& Wiesel, T. N. Functional architecture of macaque monkey striate cortex. Proceedings of the Royal Society, London (Series B), 1977, 198, 1-59.

InDow, T. Alleys in visual space. Journal of Mathematical Psychology, 1979, 19, 221-258.

LUNEBURg, R. K. Mathematical analysis of binocular vision. Princeton, N.J: Princeton University Press, 1947.

MacKAY, D. M. Interactive process in visual perception. In W. A. Rosenblith (Ed.), Sensory communication. New York: Wiley, 1961.

MARR, D. Early processing of visual information. Philosophical Transactions of the Royal Society, London (Series B), 1976, 275, 483-524.

Paillard, J. The Lie transformation group model for perceptual and cognitive psychology. Cahiers de Psychologie, 1977, 20, No. 2 (whole issue).

She Phe Rd, G. M. The synaptic organization of the brain. New York: Oxford University Press, 1974.

Sholl, D. A. The organization of the cerebral cortex. London: Methuen, 1956.
Wilkinson, F. E., \& Dodwell, P. C. Young kittens can learn complex pattern discriminations. Nature, 1980, 284, 258-259. Zusne, L. Visual perception of form. New York: Academic Press, 1970.

\section{NOTES}

1. The terms model and theory are used in this paper as follows: a (mathematical) theory is the abstract and symbolic expression of a formalism, or "uninterpreted calculus" (Campbell, 1920). A model of that theory is its embodiment in some real mechanical, electronic, biological, or other system. The term "theory" is also used in a more general sense; one can hold the theory that a particular model applies in some given situation. Thus, Hoffman holds the theory that the LTG model applies to visual processing.

2. "Computing an invariance" does not necessarily entail the sort of explicit computation that might be carried out in a digital machine. It could, for example, be embodied in an analog process that "matches" or "resonates to" a particular input pattern. See section VI below.

3. Under some reasonable assumptions, it can be shown that $\mathscr{\mathscr { L }}_{\mathrm{R}}=-\mathrm{y}(\partial / \partial \mathrm{x})+\mathrm{x}(\partial \mathrm{L} \partial \mathrm{y})$ implies $-\mathrm{y}(\mathrm{dy} / \mathrm{dx})+\mathrm{x}=0$, or $\mathrm{dy} / \mathrm{dx}=$ $(-x / y)$, which was shown earlier to be the differential equation generating the circular vectorfield of Figure 3.

4. In general, the first prolongation of a basic Lie operator, $\mathscr{P}^{(1)}$, involves an additional variable, $\mathrm{y}^{\prime}$, so its transformation group can be characterized by $\mathscr{T}^{(1)}=\mathscr{L}+\mathscr{L}\left(\mathrm{x}, \mathrm{y}, \mathrm{y}^{\prime}\right)(\partial / \partial \mathrm{y})$, etc. The expression $\mathscr{L}{ }^{(2)}$ for the second prolongation of $\mathscr{L}_{1}$ is $\mathrm{y}(\partial / \partial \mathrm{y})+$ $y^{\prime}\left(\partial / \partial y^{\prime}\right)+y^{\prime \prime}\left(\partial / \partial y^{\prime \prime}\right)$, where $y^{\prime}$ and $y^{\prime \prime}$ are symbols for differentiation with respect to the independent parameter $t$.

5. See Footnote 1 for my distinction between model and two senses of the word "theory." Here I am using "theory" in its second (general) sense.

(Invited paper, accepted for publication March 28, 1983.) 